\title{
Duffin-Kemmer-Petiau Theory in the Causal Approach
}

\author{
J. T. Lunardi啁, L. A. Manzoni ${ }^{b}$, B. M. Pimentel ${ }^{a}$ and \\ J. S. Valverde ${ }^{a}$ \\ ${ }^{a}$ Instituto de Física Teórica \\ Universidade Estadual Paulista \\ Rua Pamplona 145 \\ 01405-900 - São Paulo, SP \\ Brazil \\ ${ }^{b}$ Instituto de Física \\ Universidade de São Paulo \\ Caixa Postal 66318 \\ 05315-970 - São Paulo - SP \\ Brazil.
}

\begin{abstract}
In this paper we consider the scalar sector of Duffin-Kemmer-Petiau theory in the framework of Epstein-Glaser causal method. We calculate the lowest order distributions for Compton scattering, vacuum polarization, self-energy and vertex corrections. By requiring gauge invariance of the theory we recover, in a natural way, the scalar propagator of the usual effective theory.
\end{abstract}

*On leave from Universidade Estadual de Ponta Grossa, Setor de Ciências Exatas e Naturais, Departamento de Matemática e Estatística. Ponta Grossa-PR, Brazil. 


\section{Introduction}

The usual way to approach scalar quantum electrodynamics (SQED) is by performing the electromagnetic minimal coupling in the free Lagrangian of KleinGordon (KG) scalar field theory [1]. An alternative way is to start from the free Duffin-Kemmer-Petiau (DKP) Lagrangian instead of the KG one.

The free DKP theory is a theory for scalar and vector fields [2, 3, 4, 5, 6] given by a Lagrangian formally similar to that of spinorial QED. The fundamental differences are the algebraic relations satisfied by the $\beta^{\mu}$ matrices in DKP theory, which play the role analogous to $\gamma^{\mu}$ in spinor QED.

It is known that in the free field case the DKP and KG theories are equivalent, both in classical and quantum pictures [1, 7, 8, 9. However, there are still no general proofs of equivalence between these theories when interactions and decays of unstable particles are taken into account [10, 11] (in this context see also references [12, 13], which relate different results by using both DKP and KG formalisms with strong interactions). Some progress in this direction has been made recently. For instance, it was shown that both theories are equivalent in the classical level for the cases of minimal interaction with electromagnetic [8, 14] and gravitational [15] fields. Strict proofs of equivalence between both theories were also given for the cases of interaction of the quantized scalar field with classical and quantized electromagnetic, Yang-Mills and external gravitational fields [11, 16].

Perhaps one of the most evident advantages in working with this theory is the fact that derivative couplings do not appear between DKP and the gauge field (this property has been used by Gribov recently, who employed the vector sector of DKP theory to study the quark confinement problem [17]). Such property will result in manifestly covariant expressions for the interaction Hamiltonian and the 
vacuum expectation values of time ordered products of fields. Another advantages are the formal similarity with spinor QED (which facilitates the adaptation to the scalar case of technics developed formerly in the spinor case [12, 18]) and the fact that this formalism allows an unified treatment of the scalar and vector fields.

One of the difficulties in working with SQED based on KG equation (SQED$\mathrm{KG}$ ) is the presence of a term of second order in the coupling constant in the interaction Hamiltonian, which causes trouble in proving gauge invariance [19]. In SQED based on DKP theory it was achieved, by an effective approach, that this second order term does not contribute to S-matrix, and thus it can be neglected when we construct the Feynman rules for the theory [9, 16].

In this paper we will consider SQED-DKP in the framework of Epstein-Glaser causal perturbative method. This method was formulated to give a mathematical rigorous treatment of ultraviolet divergences in quantum field theory. In this framework such divergences do not appear anywhere in the calculations due to the correct splitting of causal distributions into its advanced and retarded parts [20, 21]. Our goals are to obtain a non-effective and mathematically well defined theory for SQED-DKP and recover the results of the corresponding effective theory already obtained in the usual perturbative approach. In addition, this work must be viewed as an initial step in the attempts to rigorously establish the renormalizability of the theory which is still an open question.

This paper is organized as follows. In section 2 we present the DKP theory. In section 3 we review the main features of the Epstein-Glaser causal method and present the basis to construct the second order S matrix. In section 4 we consider Compton scattering and address the question of how the propagator of the effective theory emerges in the causal approach. In this context the gauge invariance plays a crucial role. In section 5 we calculate the scalar vacuum polar- 
ization tensor. In section 6 we calculate the self-energy and, by using an Ward identity, also the vertex correction in the limit of zero momentum transfer. In section 7 we make our concluding remarks. The implications of gauge invariance are presented in the Appendix.

\section{Duffin-Kemmer-Petiau theory}

The free DKP theory is given by the Lagrangian [6, 8, 9]

$$
\mathcal{L}=\frac{i}{2} \bar{\psi} \beta^{\mu} \overleftrightarrow{\partial}_{\mu} \psi-m \bar{\psi} \psi
$$

where $\psi$ is a multicomponent wave function, $\bar{\psi}=\psi^{\dagger} \eta^{0}$, and $\eta^{0}=2\left(\beta^{0}\right)^{2}-1$. $\beta^{\mu}$

are a set of matrices $(\mu=0,1,2,3)$ satisfying the algebraic relations

$$
\beta^{\mu} \beta^{\nu} \beta^{\rho}+\beta^{\rho} \beta^{\nu} \beta^{\mu}=\beta^{\mu} g^{\nu \rho}+\beta^{\rho} g^{\mu \nu}
$$

The equations of motion are then

$$
\left(i \beta^{\mu} \partial_{\nu}-m\right) \psi=0 \quad \text { and } \quad \bar{\psi}\left(i \beta^{\mu} \partial_{\nu}+m\right)=0
$$

It is known 22] that the algebra (2) has only three irreducible representations, whose degrees are 1,5 and 10. The first one is trivial, having no physical content. The second and the third ones correspond, respectively, to the scalar and vectorial representations. In this work we shall restrict us to the scalar case.

Defining $\not=\beta^{\mu} q_{\mu}$ and using relations (2) it can be shown that, for any fourvector $q$ the following relation is satisfied

$$
\not q^{2}\left(\not q^{2}-q^{2}\right)=0
$$

By using this relation and the plane wave solutions for the equations of motion we can verify that $p^{2}=m^{2}$. 
As an ilustrative example of an explicit representation of (2) we can get

$$
\begin{aligned}
\beta^{0}= & \left(\begin{array}{ccccc}
0 & 0 & 0 & 0 & 1 \\
0 & 0 & 0 & 0 & 0 \\
0 & 0 & 0 & 0 & 0 \\
0 & 0 & 0 & 0 & 0 \\
1 & 0 & 0 & 0 & 0
\end{array}\right) ; \quad \beta^{1}=\left(\begin{array}{ccccc}
0 & 0 & 0 & 0 & 0 \\
0 & 0 & 0 & 0 & 1 \\
0 & 0 & 0 & 0 & 0 \\
0 & 0 & 0 & 0 & 0 \\
0 & -1 & 0 & 0 & 0
\end{array}\right) ; \\
\beta^{2}= & \left(\begin{array}{ccccc}
0 & 0 & 0 & 0 & 0 \\
0 & 0 & 0 & 0 & 0 \\
0 & 0 & 0 & 0 & 1 \\
0 & 0 & 0 & 0 & 0 \\
0 & 0 & -1 & 0 & 0
\end{array}\right) ; \quad \beta^{3}=\left(\begin{array}{ccccc}
0 & 0 & 0 & 0 & 0 \\
0 & 0 & 0 & 0 & 0 \\
0 & 0 & 0 & 0 & 0 \\
0 & 0 & 0 & 0 & 1 \\
0 & 0 & 0 & -1 & 0
\end{array}\right) .
\end{aligned}
$$

Using this specific representation we can construct explicitly projection operators and show that not all components of the field $\psi$ are independent. For a detailed explanation we refer to references [6, 8]. Here we only quote the explicit form of $\psi$ in this representation:

$$
\psi=\left(\begin{array}{c}
\frac{i}{\sqrt{m}} \partial_{0} \varphi \\
\frac{i}{\sqrt{m}} \partial_{1} \varphi \\
\frac{i}{\sqrt{m}} \partial_{2} \varphi \\
\frac{i}{\sqrt{m}} \partial_{3} \varphi \\
\sqrt{m} \varphi
\end{array}\right), \quad\left(\square+m^{2}\right) \varphi=0
$$

Thus we readily see that not all components of $\psi$ can be independent. Actually, we can choose only two of these components as independent ones. We shall make use of this explicit representation in the remainder of this work only for ilustrating the arguments, being our results valid for any scalar representation of relations (2). 
Now we apply the standard procedure of canonical quantization in the free Lagrangian (11) and obtain [9]

$$
\left[\psi_{a}^{-}(x),{\overline{\psi^{+}}}_{b}(y)\right]=\frac{1}{i} S_{a b}^{+}(x-y) \quad \text { and } \quad\left[{\overline{\psi^{-}}}_{a}(x), \psi_{b}^{+}(y)\right]=-\frac{1}{i} S_{b a}^{-}(y-x)
$$

where $\psi^{-}$and $\psi^{+}$contains only annihilation and creation operators, respectively and

$$
S_{a b}^{ \pm}(x)=\frac{1}{m}[i \not \partial(i \not \partial+m)]_{a b} \triangle^{ \pm}(x),
$$

where

$$
\triangle^{ \pm}(x)=\frac{( \pm) i}{(2 \pi)^{3}} \int d^{4} p \delta\left(p^{2}-m^{2}\right) \theta\left( \pm p^{0}\right) \mathrm{e}^{-i p \cdot x}
$$

are the positive (negative) frequency parts of the Pauli-Jordan distribution

$$
\triangle(x)=\triangle^{+}(x)+\triangle^{-}(x)
$$

As it is well known, this later distribution has causal support [21] and can be written as

$$
\triangle(x)=\triangle^{\mathrm{ret}}(x)-\triangle^{\mathrm{adv}}(x),
$$

where $\triangle^{\text {ret }}(x)$ and $\triangle^{\text {adv }}(x)$ have, respectively, retarded and advanced supports with respect to the point $x$. Analogously we define

$$
S(x) \triangleq S^{+}(x)+S^{-}(x)
$$

We can see directly that this distribution also has a causal support, since it is a linear combination of derivatives of $\triangle(x)$, and the differentiation of a causal distribution does not affect the causal property of its support. Now, by (7) and (9), it is possible to write

$$
S(x)=S^{\mathrm{ret}}(x)-S^{\mathrm{adv}}(x)
$$

where

$$
S^{\mathrm{ret}}(x)=\frac{1}{m}[i \not \partial(i \not \partial+m)] \triangle^{\mathrm{ret}}(x) \quad \text { and } \quad S^{\mathrm{adv}}(x)=\frac{1}{m}[i \not \partial(i \not \partial+m)] \triangle^{\mathrm{adv}}(x) .
$$


As we will see in the next section, the above splitting of $S(x)$ in retarded and advanced parts is not the unique possible.

The interaction with electromagnetic field is introduced by the minimal substitution $\partial_{\mu} \rightarrow \partial_{\mu}-i e A_{\mu}$ in the Lagrangian (1), which becomes

$$
\begin{aligned}
\mathcal{L} & =\mathcal{L}_{\mathrm{F}}+\mathcal{L}_{\mathrm{I}} ; \\
\mathcal{L}_{\mathrm{F}} & =\frac{i}{2} \bar{\psi} \beta^{\mu} \overleftrightarrow{\partial_{\mu}} \psi-m \bar{\psi} \psi ; \\
\mathcal{L}_{\mathrm{I}} & =e \bar{\psi} \beta^{\mu} \psi A_{\mu},
\end{aligned}
$$

where we have used $e>0$.

\section{The Epstein-Glaser causal approach}

In the Epstein-Glaser's causal method [20] the $S$-matrix is constructed without any reference to Hamiltonian formalism, its explicit form being obtained by making use of certain physical conditions - with causality playing a major role. In this approach the S-matrix is viewed as an operator-valued distribution given by the perturbative series

$$
S(g)=1+\sum_{n=1}^{\infty} \frac{1}{n !} \int d^{4} x_{1} \ldots d^{4} x_{n} T_{n}\left(x_{1}, \ldots, x_{n}\right) g\left(x_{1}\right) \ldots g\left(x_{n}\right),
$$

where $g(x)$ is a c-number test function supposed to belong to the Schwartz space, $g(x) \in \mathcal{S}\left(\mathbf{R}^{4}\right)$. The symmetric $n$-point functions $T_{n}(X)\left(X \equiv\left\{x_{1}, \ldots, x_{n}\right\}\right)$ are the basic building blocks to be inductively constructed, from the knowledge of $T_{1}(x)$, by means of the requirements of causality

$$
S\left(g_{1}+g_{2}\right)=S\left(g_{1}\right) S\left(g_{2}\right), \quad \text { if } \operatorname{supp} g_{1}>\operatorname{supp} g_{2},
$$


and translational invariance

$$
U(a, \mathbf{1}) S(g) U(a, \mathbf{1})^{-1}=S(g(x-a))
$$

In the above equations $U(a, \mathbf{1})$ is an usual representation of the Poincaré group $\mathcal{P}_{+}^{\uparrow}$ in the Fock space and the notation supp $g_{1}>\operatorname{supp} g_{2}$ signify that all points in the support of $g_{2}(x)$ occur at times previous than all points in the support of $g_{1}(x)$.

In terms of the $n$-point functions $T_{n}$ these causality and translation invariance conditions are given by

$$
\begin{array}{r}
T_{n}\left(x_{1}, \ldots, x_{n}\right)=\quad T_{m}\left(x_{1}, \ldots, x_{m}\right) T_{n-m}\left(x_{m+1}, \ldots, x_{n}\right), \\
\text { if } \quad\left\{x_{1}, \ldots, x_{m}\right\}>\left\{x_{m+1}, \ldots, x_{n}\right\},
\end{array}
$$

and

$$
U(a, \mathbf{1}) T_{n}\left(x_{1}, \ldots, x_{n}\right) U(a, \mathbf{1})^{-1}=T_{n}\left(x_{1}+a, \ldots, x_{n}+a\right),
$$

respectively.

Making use of these requirements we are able to construct the $T_{n}$, order by order, from the explicit form of $T_{1}(x)$. It is known that, based on general arguments such as correspondence [7], $T_{1}=i \mathcal{L}_{I}^{(1)}$, where $\mathcal{L}_{I}^{(1)}$ is the term of first order in coupling constant in the interaction Lagrangian and is written in terms of free fields [19].

Now, let us sketch the inductive procedure (for a detailed account see [21, 23]). Suppose that all $T_{m}(X)$, with $m \leq n-1$ are known, then one can define the distributions

$$
A_{n}^{\prime}\left(x_{1}, \ldots, x_{n}\right)=\sum_{P_{2}} \tilde{T}_{n_{1}}(X) T_{n-n_{1}}\left(Y, x_{n}\right),
$$




$$
R_{n}^{\prime}\left(x_{1}, \ldots, x_{n}\right)=\sum_{P_{2}} T_{n-n_{1}}\left(Y, x_{n}\right) \tilde{T}_{n_{1}}(X)
$$

where $P_{2}$ stands for all partitions $P_{2}:\left\{x_{1}, \ldots, x_{n-1}\right\}=X \cup Y, X \neq \varnothing$ into disjoint subsets with $|X|=n_{1},|Y| \leq n-2$. In (21) $\tilde{T}_{n}(X)$ refers to the $n$-point distributions corresponding to a series for the $S^{-1}$-matrix analogous to (16) and can be obtained by formal inversion of $S(g)$, giving

$$
\tilde{T}_{n}(X)=\sum_{r=1}^{n}(-)^{r} \sum_{P_{r}} T_{n_{1}}\left(X_{1}\right) \ldots T_{n_{r}}\left(X_{r}\right),
$$

where $P_{r}$ indicates all partitions of $X$ into $r$ disjoint subsets: $X=X_{1} \cup \ldots \cup X_{r}$, $X_{j} \neq \varnothing,\left|X_{j}\right|=n_{j}$. Of course, since all $T_{m}(X), m \leq n-1$ are given by the induction hypothesis, also the $\tilde{T}_{m}(X)$ with $m \leq n-1$ are known.

If in (21) the sums are extended in order to include the empty set $X=\varnothing$ we get

$$
\begin{aligned}
A_{n}\left(x_{1}, \ldots, x_{n}\right) & =\sum_{P_{2}^{0}} \tilde{T}_{n_{1}}(X) T_{n-n_{1}}\left(Y, x_{n}\right) \\
& =A_{n}^{\prime}\left(x_{1}, \ldots, x_{n}\right)+T_{n}\left(x_{1}, \ldots, x_{n}\right), \\
R_{n}\left(x_{1}, \ldots, x_{n}\right) & =\sum_{P_{2}^{0}} T_{n-n_{1}}\left(Y, x_{n}\right) \tilde{T}_{n_{1}}(X) \\
& =R_{n}^{\prime}\left(x_{1}, \ldots, x_{n}\right)+T_{n}\left(x_{1}, \ldots, x_{n}\right),
\end{aligned}
$$

where $P_{2}^{0}$ stands for all partitions $P_{2}^{0}:\left\{x_{1}, \ldots, x_{n-1}\right\}=X \cup Y$. A glance at equations (23) shows that $A_{n}$ and $R_{n}$ are not known because they contain the unknown $T_{n}$. However, the distribution defined by 


$$
D_{n}\left(x_{1}, \ldots, x_{n}\right) \equiv R_{n}-A_{n}=R_{n}^{\prime}-A_{n}^{\prime}
$$

is known.

Making use of causality it turns out that $R_{n}$ has retarded support and $A_{n}$ has advanced support, i.e.

$$
\operatorname{supp} R_{n}(X) \subseteq \Gamma_{n-1}^{+}\left(x_{n}\right), \quad \operatorname{supp} A_{n}(X) \subseteq \Gamma_{n-1}^{-}\left(x_{n}\right),
$$

with

$$
\begin{array}{r}
\Gamma_{n-1}^{ \pm}(x) \equiv\left\{\left(x_{1}, \ldots, x_{n-1}\right) \mid x_{j} \in \bar{V}^{ \pm}(x), \forall j=1, \ldots, n-1\right\}, \\
\bar{V}^{ \pm}(x)=\left\{y \mid(y-x)^{2} \geq 0, \pm\left(y^{0}-x^{0}\right) \geq 0\right\} .
\end{array}
$$

The distribution $D_{n}$ has causal support, $\operatorname{supp} D_{n} \subseteq \Gamma_{n-1}^{+} \cup \Gamma_{n-1}^{-}$. In fact, a general proof of the causal support of $D_{n}$ only exists for $n \geq 3$ - for $n=2$ we must verify this explicitly. Then, decomposing $D_{n}$ in advanced and retarded distributions we obtain the $T_{n}$ distribution by (23).

The operator-valued distributions which we shall have to split are of the form

$$
D_{n}\left(x_{1}, \ldots, x_{n}\right)=\sum_{k}: \prod_{j} \bar{\psi}\left(x_{j}\right) d_{n}^{k}\left(x_{1}, \ldots, x_{n}\right) \prod_{l} \psi\left(x_{l}\right) \prod_{m} A\left(x_{m}\right):
$$

where $\psi, \bar{\psi}$ are the free boson fields of DKP theory and $A$ stands for the free gauge boson fields. In this expression $d_{n}^{k}$ are numerical tempered distributions, $d_{n}^{k} \in \mathcal{S}^{\prime}\left(\mathbf{R}^{\mathbf{4 n}}\right)$, with causal support. Because of the translation invariance, it is sufficient to put $x_{n}=0$ and consider 


$$
d(x) \equiv d_{n}^{k}\left(x_{1}, \ldots, x_{n-1}, 0\right) \in \mathcal{S}^{\prime}\left(\mathbf{R}^{\mathbf{m}}\right), \quad \mathbf{m}=4 \mathbf{n}-4
$$

The nontrivial step is the splitting of the numerical causal distribution $d$ in the advanced and retarded distributions $a$ and $r$, respectively. From the fact that $\Gamma^{+}(0) \cap \Gamma^{-}(0)=\{0\}$ we can see that the behaviour of $d(x)$ in $x=0$ (or, in momentum space, $p=\infty$ ) is crucial in the splitting problem. With this in mind, a classification of the distributions is given in which $d(x) \in \mathcal{S}^{\prime}\left(\mathbf{R}^{\mathbf{m}}\right)$ is called singular of order $\omega$ if its Fourier transform $\hat{d}(p)$ has a quasi-asymptotics $\hat{d}_{0}(p)$ at $p=\infty$ 21, 23,

$$
\lim _{\delta \rightarrow 0} \rho(\delta)\left\langle\hat{d}\left(\frac{p}{\delta}\right), \stackrel{\vee}{\phi}(p)\right\rangle=\left\langle\hat{d}_{0}, \stackrel{\vee}{\phi}\right\rangle
$$

$(\phi$ is the inverse Fourier transform of $\phi)$, with power-counting function $\rho(\delta)$ satisfying

$$
\lim _{\delta \rightarrow 0} \frac{\rho(c \delta)}{\rho(\delta)}=c^{\omega}
$$

for each $c>0$. Of course, there is an equivalent definition in the coordinate space [21], but, since the splitting is more easily performed in the momentum space, this one is sufficient for our purposes.

Then, we have two distinct cases [20, 21]: i) $\omega<0$ - in this case the solution of the splitting problem is unique and the retarded distribution can be defined by multiplication by step functions; ii) $\omega \geq 0$ - now the solution can be no longer obtained by multiplying $d$ by step functions and, after a careful mathematical treatment, it may be shown that the retarded distribution is given by the "central splitting solution" 21

$$
\hat{r}(p)=\frac{i}{2 \pi} \int_{-\infty}^{+\infty} d t \frac{\hat{d}(t p)}{(t-i 0)^{\omega+1}(1-t+i 0)} .
$$


This solution has the very important feature that it preserves the symmetries of the theory, in special Lorentz covariance and gauge invariance. However, in contrast with the case $\omega<0$, the solution of the splitting problem (31) is not the unique one and, in momentum space, the general solution is given by

$$
\tilde{r}(p)=\hat{r}(p)+\sum_{|a|=0}^{\omega} C_{a} p^{a},
$$

where the $C_{a}$ are constant coefficients which are not fixed by the causal structure - we need additional physical conditions in order to determine them.

In expression (32) use is made of the minimal distribution splitting condition which says that the singular order cannot be raised in the splitting. This condition, crucial for a correct prediction of the anomalous magnetic moment in $\mathrm{QED}_{4}$ [21] and in the analysis of the dynamic mass generation in $(2+1)$ dimensions [24, 25], will also be useful here.

Now we apply the inductive steps above to construct the two-point distributions for SQED-DKP theory. Then, the one-point distribution for the DKP field interacting with electromagnetic field is given formally by $i$ times the interaction term (15) in the Lagrangian (13),

$$
T_{1}(x)=i e: \bar{\psi}(x) \beta^{\mu} \psi(x): A_{\mu}(x)=-\widetilde{T}_{1}(x)
$$

where all fields entering in this expression are free fields and $e$ is the physical charge. The normal ordering is necessary in order to have a well defined expression for the product of field operators at the same point.

To go from $n=1$ to $n=2$ we take (33) and construct the distributions $A_{2}^{\prime}\left(x_{1}, x_{2}\right)$ and $R_{2}^{\prime}\left(x_{1}, x_{2}\right)$ from expressions (23). So, we have

$$
\begin{aligned}
& A_{2}^{\prime}\left(x_{1}, x_{2}\right)=e^{2}: \bar{\psi}\left(x_{1}\right) \beta^{\mu} \psi\left(x_{1}\right):: \bar{\psi}\left(x_{2}\right) \beta^{\nu} \psi\left(x_{2}\right): A_{\mu}\left(x_{1}\right) A_{\nu}\left(x_{2}\right) \\
& R_{2}^{\prime}\left(x_{1}, x_{2}\right)=e^{2}: \bar{\psi}\left(x_{2}\right) \beta^{\nu} \psi\left(x_{2}\right):: \bar{\psi}\left(x_{1}\right) \beta^{\mu} \psi\left(x_{1}\right): A_{\mu}\left(x_{1}\right) A_{\nu}\left(x_{2}\right)
\end{aligned}
$$


and then

$$
\begin{aligned}
D_{2}\left(x_{1}, x_{2}\right)= & e^{2}\left\{: \bar{\psi}\left(x_{2}\right) \beta^{\nu} \psi\left(x_{2}\right):: \bar{\psi}\left(x_{1}\right) \beta^{\mu} \psi\left(x_{1}\right):\right. \\
& \left.-: \bar{\psi}\left(x_{1}\right) \beta^{\mu} \psi\left(x_{1}\right):: \bar{\psi}\left(x_{2}\right) \beta^{\nu} \psi\left(x_{2}\right):\right\} A_{\mu}\left(x_{1}\right) A_{\nu}\left(x_{2}\right)
\end{aligned}
$$

By using Wick's Theorem this expression can be written as a sum of terms, each of them consisting of a product of field operators and Wick contractions [21]. In this work we shall be concerned to the terms corresponding to Compton scattering, vacuum polarization and self-energy. From these we will determine the vertex correction via an Ward identity. These terms are given by

$$
\begin{aligned}
& D_{2}^{\text {Com }}\left(x_{1}, x_{2}\right)=e^{2} \beta_{a b}^{\mu} \beta_{c d}^{\nu} \\
& \times\{: \bar{\psi}_{a}\left(x_{1}\right) \psi_{d}\left(x_{2}\right):: A_{\mu}\left(x_{1}\right) A_{\nu}\left(x_{2}\right): \overbrace{\left[\bar{\psi}_{c}\left(x_{2}\right) \psi_{b}\right.}\left(x_{1}\right)-\overbrace{\psi_{b}\left(x_{1}\right) \bar{\psi}_{c}}\left(x_{2}\right)] \\
& +: \psi_{b}\left(x_{1}\right) \bar{\psi}_{c}\left(x_{2}\right):: A_{\mu}\left(x_{1}\right) A_{\nu}\left(x_{2}\right): \overbrace{\left[\psi_{d}\left(x_{2}\right) \bar{\psi}_{a}\right.}\left(x_{1}\right)-\overbrace{\bar{\psi}_{a}\left(x_{1}\right) \psi_{d}}\left(x_{2}\right)]\} \\
& D_{2}^{\operatorname{Vac}}\left(x_{1}, x_{2}\right)=e^{2} \beta_{a b}^{\mu} \beta_{c d}^{\nu}: A_{\mu}\left(x_{1}\right) A_{\nu}\left(x_{2}\right): \\
& \times[\overbrace{\bar{\psi}_{c}\left(x_{2}\right) \psi_{b}}\left(x_{1}\right) \overbrace{\psi_{d}\left(x_{2}\right) \bar{\psi}_{a}}\left(x_{1}\right)-\overbrace{\bar{\psi}_{a}\left(x_{1}\right) \psi_{d}}\left(x_{2}\right)], \\
& D_{2}^{\text {self }}\left(x_{1}, x_{2}\right)=e^{2} \beta_{a b}^{\mu} \beta_{c d}^{\nu} \\
& \times\{: \bar{\psi}_{a}\left(x_{1}\right) \psi_{d}\left(x_{2}\right): \overbrace{\widetilde{\psi}_{c}\left(x_{2}\right) \psi_{b}}\left(x_{1}\right) \overbrace{A_{\nu}\left(x_{2}\right) A_{\mu}}\left(x_{1}\right) \\
& -\overbrace{\psi_{b}\left(x_{1}\right) \bar{\psi}_{c}}\left(x_{2}\right) \overbrace{A_{\mu}\left(x_{1}\right) A_{\nu}}\left(x_{2}\right)] \\
& +: \psi_{b}\left(x_{1}\right) \bar{\psi}_{c}\left(x_{2}\right): \overbrace{\left[\psi_{d}\left(x_{2}\right) \bar{\psi}_{a}\right.}\left(x_{1}\right) \overbrace{A_{\nu}\left(x_{2}\right) A_{\mu}}\left(x_{1}\right) \\
& -\overbrace{\bar{\psi}_{a}\left(x_{1}\right) \psi_{d}}\left(x_{2}\right) \overbrace{A_{\mu}\left(x_{1}\right) A_{\nu}}\left(x_{2}\right)]\}
\end{aligned}
$$

where the Wick contractions are defined as

$$
\begin{aligned}
& \overbrace{\psi_{a}(x) \bar{\psi}_{b}}(y) \triangleq\left[\psi_{a}^{-}(x), \bar{\psi}_{b}^{+}(y)\right]=\frac{1}{i} S_{a b}^{+}(x-y) ; \\
& \overbrace{\bar{\psi}_{a}(x) \psi_{b}}(y) \triangleq\left[\overline{\psi_{a}^{-}}(x), \psi_{b}^{+}(y)\right]=-\frac{1}{i} S_{b a}^{-}(y-x) ;
\end{aligned}
$$




$$
\overbrace{A_{\mu}(x) A_{\nu}}(y) \triangleq\left[A_{\mu}^{-}(x), A_{\nu}^{+}(y] \quad=i g_{\mu \nu} D_{0}^{+}(x-y)\right.
$$

and $S_{a b}^{+}(x)$ and $S_{b a}^{-}(x)$ are given by (7). $D_{0}^{+}(x)$ is the positive frequency part of the zero mass Pauli-Jordan distribution, $D_{0}^{+}(x)=\frac{i}{(2 \pi)^{3}} \int d^{4} p \delta\left(p^{2}\right) \theta\left(p^{0}\right) \mathrm{e}^{-i p \cdot x}$.

\section{The Compton scattering}

We denote respectively by $D_{2}^{\mathrm{I}}\left(x_{1}, x_{2}\right)$ and $D_{2}^{\mathrm{II}}\left(x_{1}, x_{2}\right)$ the first and second terms inside curl brackets in (38). By using (41) and 42) we have

$$
\begin{aligned}
D_{2}^{\mathrm{I}}\left(x_{1}, x_{2}\right)= & i e^{2} \beta_{a b}^{\mu} \beta_{c d}^{\nu}: \bar{\psi}_{a}\left(x_{1}\right) \psi_{d}\left(x_{2}\right):: A_{\mu}\left(x_{1}\right) A_{\nu}\left(x_{2}\right): \\
& \times\left\{S_{b c}^{-}\left(x_{1}-x_{2}\right)+S_{b c}^{+}\left(x_{1}-x_{2}\right)\right\} \\
= & i e^{2} \beta_{a b}^{\mu} \beta_{c d}^{\nu}: \bar{\psi}_{a}\left(x_{1}\right) \psi_{d}\left(x_{2}\right):: A_{\mu}\left(x_{1}\right) A_{\nu}\left(x_{2}\right): S_{b c}\left(x_{1}-x_{2}\right)
\end{aligned}
$$

where we used the definition (10). The first term inside curl brackets in (44) cames from $R_{2}^{\prime \mathrm{I}}\left(x_{1}, x_{2}\right)$, whereas the second cames from $A_{2}^{\prime \mathrm{I}}\left(x_{1}, x_{2}\right)$. Similarly,

$$
\begin{aligned}
D^{\mathrm{II}}\left(x_{1}, x_{2}\right)= & i e^{2} \beta_{a b}^{\mu} \beta_{c d}^{\nu}: \psi_{b}\left(x_{1}\right) \bar{\psi}_{c}\left(x_{2}\right):: A_{\mu}\left(x_{1}\right) A_{\nu}\left(x_{2}\right): \\
& \times\left\{-S_{d a}^{+}\left(x_{2}-x_{1}\right)-S_{d a}^{-}\left(x_{2}-x_{1}\right)\right\} \\
= & i e^{2} \beta_{a b}^{\mu} \beta_{c d}^{\nu}: \psi_{b}\left(x_{1}\right) \bar{\psi}_{c}\left(x_{2}\right):: A_{\mu}\left(x_{1}\right) A_{\nu}\left(x_{2}\right): \\
& \times\left\{-S_{d a}\left(x_{2}-x_{1}\right)\right\},
\end{aligned}
$$

where again the first term inside curl brackets in (46) cames from $R_{2}^{\prime I I}\left(x_{1}, x_{2}\right)$ and so on. As we said in Section 2, the distribution $S(x)$ has causal support. Then, we have verified explicitly that the distributions (45) and (47) have causal support too.

Since $S(x)$ itself is the numerical distribution we must split into retarded and advanced parts in equations (45) and (47), a splitting solution is trivially obtained from (11). But, since $S(x)$ has singular order $\omega=0$-as we can verify by (29) and 
(30) - this splitting is not unique. So, accordingly with (32), the general solution for the retarded distribution in configuration space is

$$
\tilde{r}(x)=S^{\mathrm{ret}}(x)+C \delta(x)
$$

where $C$ is an arbitrary constant. Now we construct the numerical distribution $t^{\mathrm{I}}\left(x_{1}, x_{2}\right)$ from $(23)$,

$$
t^{\mathrm{I}}\left(x_{1}, x_{2}\right)=\tilde{r}\left(x_{1}, x_{2}\right)-r^{\prime}\left(x_{1}, x_{2}\right)
$$

where $r^{\prime}\left(x_{1}, x_{2}\right)$ is the numerical distribution associated with $R_{2}^{\prime I}\left(x_{1}, x_{2}\right)$, which is obtained from the first term inside curl brackets in (44). Then,

$$
\begin{aligned}
t^{\mathrm{I}}\left(x_{1}, x_{2}\right) & =S^{\mathrm{ret}}\left(x_{1}-x_{2}\right)-S^{-}\left(x_{1}-x_{2}\right)+C \delta\left(x_{1}-x_{2}\right) \\
& =-S^{\mathrm{F}}\left(x_{1}-x_{2}\right)+C \delta\left(x_{1}-x_{2}\right)
\end{aligned}
$$

where we have defined

$$
-S^{\mathrm{F}}(x) \triangleq S^{\mathrm{ret}}(x)-S^{-}(x)=S^{\mathrm{adv}}(x)+S^{+}(x)=-\frac{1}{m} i \not \partial(i \not \partial+m) \triangle^{\mathrm{F}}(x),
$$

where $\triangle^{\mathrm{F}}(x)$ is the usual Feynman scalar propagator.

In a similar way we find (note that the reference point for splitting is $x_{2}$ )

$$
\begin{aligned}
t^{\mathrm{II}}\left(x_{1}, x_{2}\right) & =S^{\mathrm{adv}}\left(x_{2}-x_{1}\right)+S^{+}\left(x_{2}-x_{1}\right)+C^{\prime} \delta\left(x_{2}-x_{1}\right) \\
& =-S^{\mathrm{F}}\left(x_{2}-x_{1}\right)+C^{\prime} \delta\left(x_{2}-x_{1}\right) .
\end{aligned}
$$

The constants $C$ and $C^{\prime}$ will be determined later by the requirements of charge conjugation and gauge invariance.

Now the complete two-point distribution for Compton scattering is given by

$$
T_{2}^{\text {Compton }}\left(x_{1}, x_{2}\right)=T_{2}^{\mathrm{I}}\left(x_{1}, x_{2}\right)+T_{2}^{\mathrm{II}}\left(x_{1}, x_{2}\right),
$$


where

$$
T_{2}^{\mathrm{I}}\left(x_{1}, x_{2}\right)=i e^{2}: \bar{\psi}\left(x_{1}\right) \beta^{\mu} t^{\mathrm{I}}\left(x_{1}, x_{2}\right) \beta^{\nu} \psi\left(x_{2}\right):: A_{\mu}\left(x_{1}\right) A_{\nu}\left(x_{2}\right):
$$

and

$$
T_{2}^{\mathrm{II}}\left(x_{1}, x_{2}\right)=i e^{2}: \bar{\psi}\left(x_{2}\right) \beta^{\nu} t^{\mathrm{II}}\left(x_{1}, x_{2}\right) \beta^{\mu} \psi\left(x_{1}\right):: A_{\mu}\left(x_{1}\right) A_{\nu}\left(x_{2}\right): .
$$

It is very simple to require charge conjugation invariance of the theory [9, 21]. Here we only quote the condition that arise from this invariance, namely

$$
C=C^{\prime}
$$

Thus we can immediately see that

$$
T_{2}^{\mathrm{I}}\left(x_{1}, x_{2}\right)=T_{2}^{\mathrm{II}}\left(x_{2}, x_{1}\right)
$$

what implies that $T_{2}^{\text {Compton }}\left(x_{1}, x_{2}\right)$ is symmetric in its arguments, as it would be.

In the Appendix we calculate the remaining constant $C$ by requiring gauge invariance of the theory. There we find (see eq. (90))

$$
C=\frac{I}{m}
$$

where $I$ is the $5 \times 5$ identity matrix. Turning this result into (49) and (51) we obtain

$$
\begin{aligned}
t^{\mathrm{I}}\left(x_{1}, x_{2}\right) & =-\left\{S^{\mathrm{F}}\left(x_{1}-x_{2}\right)-\frac{\hat{I}}{m} \delta\left(x_{1}-x_{2}\right)\right\} \\
& =t^{\mathrm{II}}\left(x_{2}, x_{1}\right)
\end{aligned}
$$

We denote the distribution inside the curl brackets in the above expressions as

$$
T^{\mathrm{c}}(x) \triangleq S^{\mathrm{F}}(x)-\frac{\hat{I}}{m} \delta(x)
$$

It is direct to see that this distribution is the Green function for the DKP equation, i. e.,

$$
(i \not \partial-m) T^{\mathrm{c}}(x)=\delta(x)
$$


So, in this sense the distribution (53) is the correct "propagator" for the DKP scalar particle, that is the same as the "effective" propagator of references [9, 16]. For later use, we write this propagator in the momentum space

$$
\widehat{T}^{\mathrm{c}}(p)=\frac{1}{(2 \pi)^{2} m}\left[\frac{\not p(p+m)}{p^{2}-m^{2}+i 0}-1\right] .
$$

With the above results we write finally the two-point distribution for the Compton scattering:

$$
\begin{aligned}
T_{2}^{\text {Compton }}\left(x_{1}, x_{2}\right)= & -i e^{2}\left\{: \bar{\psi}\left(x_{1}\right) \beta^{\mu} T^{\mathrm{c}}\left(x_{1}-x_{2}\right) \beta^{\nu} \psi\left(x_{2}\right):\right. \\
& \left.+: \bar{\psi}\left(x_{2}\right) \beta^{\nu} T^{\mathrm{c}}\left(x_{2}-x_{1}\right) \beta^{\mu} \psi\left(x_{1}\right):\right\}: A_{\mu}\left(x_{1}\right) A_{\nu}\left(x_{2}\right):
\end{aligned}
$$

\section{The vacuum polarization}

Now we consider the term (39), associated with scalar vacuum polarization. After substituting the explicit form of Wick contractions, this term is given by

$$
\begin{aligned}
& D_{2}^{\operatorname{Vac}}\left(x_{1}, x_{2}\right)=e^{2}: A_{\mu}\left(x_{1}\right) A_{\nu}\left(x_{2}\right): \\
& \quad \times \operatorname{Tr}\left\{\beta^{\mu} S^{-}\left(x_{1}-x_{2}\right) \beta^{\nu} S^{+}\left(x_{2}-x_{1}\right)-\beta^{\nu} S^{-}\left(x_{2}-x_{1}\right) \beta^{\mu} S^{+}\left(x_{1}-x_{2}\right)\right\}
\end{aligned}
$$

where the first term inside curl brackets cames from $R^{\prime}\left(x_{1}-x_{2}\right)$ and the second from $A^{\prime}\left(x_{1}-x_{2}\right)$. Making $y=x_{1}-x_{2}$ and defining

$$
P^{\mu \nu}(y) \triangleq-e^{2} \operatorname{Tr}\left\{\beta^{\mu} S^{+}(y) \beta^{\nu} S^{-}(-y)\right\}
$$

we write (57) in the form

$$
D_{2}^{\mathrm{Vac}}\left(x_{1}, x_{2}\right)=\left\{P^{\mu \nu}(y)-P^{\nu \mu}(-y)\right\}: A_{\mu}\left(x_{1}\right) A_{\nu}\left(x_{2}\right):
$$

This distribution has a causal support, as required. To see this we use the fact that $S(x)$ has a causal support and write the term inside curl brackets in the 
above expression as

$$
\beta^{\mu}\left[S(y) \beta^{\nu} S^{+}(-y)-S^{+}(-y) \beta^{\nu} S(-y)\right]
$$

Before splitting the causal distribution above, we consider the Fourier transform of $P^{\mu \nu}(y)$,

$$
\begin{aligned}
\hat{P}^{\mu \nu}(k) & =\frac{1}{(2 \pi)^{2}} \int d y P^{\mu \nu}(y) \mathrm{e}^{i k \cdot y} \\
& =\frac{-e^{2}}{(2 \pi)^{2}} \operatorname{Tr} \int d y \beta^{\mu} S^{+}(y) \beta^{\nu} S^{-}(-y) \mathrm{e}^{i k \cdot y}
\end{aligned}
$$

Substituting into this expression the explicit forms of $S^{+}(y)$ and $S^{-}(-y)$ given by (17) and (8), we have

$$
\begin{aligned}
\hat{P}^{\mu \nu}(k)= & \frac{-e^{2}}{(2 \pi)^{4} m^{2}} \int d p \operatorname{Tr}\left\{\beta^{\mu} \not p(\not p+m) \beta^{\nu}(\not p-\not p)(\not p-\not p-m)\right\} \\
& \times \theta\left(p^{0}\right) \delta\left(p^{2}-m^{2}\right) \theta\left(k^{0}-p^{0}\right) \delta\left[(k-p)^{2}-m^{2}\right] .
\end{aligned}
$$

To compute the trace inside this integral we need the following trace properties 26]:

$$
\begin{aligned}
\operatorname{Tr}\left\{\beta^{\mu_{1}} \beta^{\mu_{2}} \ldots \beta^{\mu_{2 n-1}}\right\} & =0 \\
\operatorname{Tr}\left\{\beta^{\mu_{1}} \beta^{\mu_{2}} \ldots \beta^{\mu_{2 n}}\right\} & =g^{\mu_{1} \mu_{2}} g^{\mu_{3} \mu_{4}} \ldots g^{\mu_{2 n-1} \mu_{2 n}}+g^{\mu_{2} \mu_{3}} g^{\mu_{4} \mu_{5}} \ldots g^{\mu_{2 n} \mu_{1}}
\end{aligned}
$$

with $n=1,2,3, \ldots$. So, we obtain

$$
\operatorname{Tr}\left\{\beta^{\mu} \not p(\not p+m) \beta^{\nu}(\not p-\not p)(\not p-\not p-m)\right\}=m^{2}\left[4 p^{\mu} p^{\nu}-2\left(k^{\mu} p^{\nu}+p^{\mu} k^{\nu}\right)+k^{\mu} k^{\nu}\right]
$$

When substituting this result into (59), and taking into account the distribution $\delta\left[(k-p)^{2}-m^{2}\right]$, we observe that $\hat{P}^{\mu \nu}(k)$ is a second rank symmetric tensor which satisfies

$$
k_{\mu} \hat{P}^{\mu \nu}(k)=0
$$


what means that the vacuum polarization term (58) is gauge invariant. Using this fact, we can write, as usual,

$$
\hat{P}^{\mu \nu}(k)=\left(k^{\mu} k^{\nu}-k^{2} g^{\mu \nu}\right) B\left(k^{2}\right),
$$

where

$$
B\left(k^{2}\right)=\frac{-1}{3 k^{2}} \hat{P}_{\mu}^{\mu}(k) .
$$

The computation of $\hat{P}_{\mu}^{\mu}(k)$ is straightforward and the final result for $\hat{P}^{\mu \nu}(k)$ is

$$
\hat{P}^{\mu \nu}(k)=\frac{-e^{2}}{(2 \pi)^{4}}\left(\frac{k^{\mu} k^{\nu}}{k^{2}}-g^{\mu \nu}\right) \frac{\pi k^{2}}{6}\left(1-\frac{4 m^{2}}{k^{2}}\right)^{\frac{3}{2}} \theta\left(k^{2}-4 m^{2}\right) \theta\left(k^{0}\right) .
$$

Now, in momentum space the numerical distribution associated with (58), which we must split, is given by

$$
\hat{P}^{\mu \nu}(k)-\hat{P}^{\mu \nu}(-k)=\frac{-e^{2}}{(2 \pi)^{4}}\left(\frac{k^{\mu} k^{\nu}}{k^{2}}-g^{\mu \nu}\right) \hat{d}(k),
$$

where

$$
\hat{d}(k)=\frac{\pi k^{2}}{6}\left(1-\frac{4 m^{2}}{k^{2}}\right)^{\frac{3}{2}} \theta\left(k^{2}-4 m^{2}\right) \operatorname{sgn}\left(k^{0}\right) .
$$

As the tensor character of (61) does not matter in its splitting procedure, the problem reduces to the splitting of $\hat{d}(k)$. To do this, we first determine the singular order $\omega$ of this distribution by using (29) and (30). So, we find $\omega=2$. To simplify the calculations we make use of the fact that $k$ in (62) is timelike. So, we can choose a Lorentz frame such that $k=\left(k^{0}, \overrightarrow{0}\right)$. In this case the central splitting formula (31) can be written as 21]:

$$
\hat{r}\left(k^{0}\right)=\frac{i}{2 \pi}\left(k^{0}\right)^{3} \int_{-\infty}^{+\infty} d p^{0} \frac{\hat{d}\left(p^{0}\right)}{\left(p^{0}-i 0\right)^{3}\left(k^{0}-p^{0}+i 0\right)} .
$$

Substituting (62) into this integral and making the substitution $s=\left(p^{0}\right)^{2}$, we obtain

$$
\hat{r}\left(k^{0}\right)=\frac{i}{12}\left(k^{0}\right)^{4}\left\{\mathrm{P} . \mathrm{V} \cdot \int_{4 m^{2}}^{\infty} d s \frac{1}{s\left[\left(k^{0}\right)^{2}-s\right]}\left(1-\frac{4 m^{2}}{s}\right)^{\frac{3}{2}}\right.
$$




$$
\left.-i \pi \operatorname{sgn}\left(k^{0}\right) \theta\left[\left(k^{0}\right)^{2}-4 m^{2}\right] \frac{1}{\left(k^{0}\right)^{2}}\left(1-\frac{4 m^{2}}{\left(k^{0}\right)^{2}}\right)^{\frac{3}{2}}\right\} .
$$

The distribution $\hat{r}^{\prime}\left(k^{0}\right)$ comes from the term $-\hat{P}^{\mu \nu}(-k)$ in (58). So,

$$
\hat{r}^{\prime}\left(k^{0}\right)=-\frac{\pi\left(k^{0}\right)^{2}}{6}\left(1-\frac{4 m^{2}}{\left(k^{0}\right)^{2}}\right)^{\frac{3}{2}} \theta\left[\left(k^{0}\right)^{2}-4 m^{2}\right] \theta\left(-k^{0}\right) .
$$

Now the two-point distribution is given, in an arbitrary Lorentz frame, as

$$
\begin{aligned}
\hat{t}(k) & =\hat{r}(k)-\hat{r}^{\prime}(k) \\
& =\frac{i}{12} k^{4} \int_{4 m^{2}}^{\infty} d s \frac{1}{s\left(k^{2}-s+i 0\right)}\left(1-\frac{4 m^{2}}{s}\right)^{\frac{3}{2}} .
\end{aligned}
$$

Finally, the two-point distribution for the vacuum polarization in configuration space is given by

$$
T_{2}^{\mathrm{Vac}}\left(x_{1}, x_{2}\right)=-i: A_{\mu}\left(x_{1}\right) \Pi^{\mu \nu}\left(x_{1}-x_{2}\right) A_{\nu}\left(x_{2}\right):
$$

where

$$
\begin{aligned}
\hat{\Pi}^{\mu \nu}(k) & =\frac{-i e^{2}}{(2 \pi)^{4}}\left(\frac{k^{\mu} k^{\nu}}{k^{2}}-g^{\mu \nu}\right) \hat{t}(k) \\
& =\frac{1}{(2 \pi)^{4}}\left(\frac{k^{\mu} k^{\nu}}{k^{2}}-g^{\mu \nu}\right) \hat{\Pi}(k)
\end{aligned}
$$

and

$$
\hat{\Pi}(k)=\frac{e^{2}}{12} k^{4} \int_{4 m^{2}}^{\infty} d s \frac{1}{s\left(k^{2}-s+i 0\right)}\left(1-\frac{4 m^{2}}{s}\right)^{\frac{3}{2}} .
$$

For $0<k^{2}<4 m^{2}$, the result of this integral can be writen as

$$
\hat{\Pi}(k)=\frac{-e^{2}}{2} k^{2}\left[\frac{4 m^{2}-k^{2}}{3 k^{2}}(\theta \cot \theta-1)+\frac{1}{9}\right],
$$

where $\sin ^{2} \theta=\frac{k^{2}}{4 m^{2}}$ and $\theta \in\left(0, \frac{\pi}{2}\right)$.

For spacelike $k$ we get

$$
\hat{\Pi}(k)=\frac{e^{2} m^{2}}{12 \lambda(1-\lambda)}\left[(1+\lambda)^{3} \ln \lambda+\frac{8}{3}\left(1-\lambda^{3}\right)\right],
$$


where $k^{2}=-\frac{(1-\lambda)^{2}}{\lambda} m^{2}$, and $0<\lambda<1$. This result can be analytically extended for $k^{2}>4 m^{2}$ by making $-1<\lambda<0$ and taking $\ln \lambda=\ln |\lambda|-i \pi$.

These results are not the most general solutions for the splitting problem, as we had to split a distribution with singular order $\omega=2$. The most general solution $\tilde{\Pi}(k)$ is given by

$$
\tilde{\Pi}(k)=\hat{\Pi}(k)+C_{0}+C_{\mu} k^{\mu}+C_{2} k^{2},
$$

where the normalization constants $C_{0}, C_{\mu}$ and $C_{2}$ are not determined by causality. They are determined from the requirements of a zero mass for the gauge field, parity invariance and the identification of $m$ with the physical observable mass. The procedure is the standard one and we simply quote the results: $C_{0}=C_{\mu}=$ $C_{2}=0$.

\section{The self-energy and vertex corrections}

The self-energy of DKP particle is given by (40). After substituting the Wick contractions we obtain the following two terms:

$$
\begin{aligned}
D_{\mathrm{I}}^{\text {self }}\left(x_{1}, x_{2}\right)= & -e^{2}: \bar{\psi}\left(x_{1}\right) \beta^{\mu}\left[S^{-}\left(x_{1}-x_{2}\right) D_{0}^{+}\left(x_{2}-x_{1}\right)\right. \\
& \left.+S^{+}\left(x_{1}-x_{2}\right) D_{0}^{+}\left(x_{1}-x_{2}\right) \beta_{\mu} \psi\left(x_{2}\right):\right] \\
D_{\mathrm{II}}^{\text {self }}\left(x_{1}, x_{2}\right)= & e^{2}: \bar{\psi}\left(x_{2}\right) \beta^{\mu}\left[S^{+}\left(x_{2}-x_{1}\right) D_{0}^{+}\left(x_{2}-x_{1}\right)\right. \\
& \left.+S^{-}\left(x_{2}-x_{1}\right) D_{0}^{+}\left(x_{1}-x_{2}\right) \beta_{\mu} \psi\left(x_{1}\right):\right]
\end{aligned}
$$

Therefore we note that

$$
D_{\mathrm{II}}^{\text {self }}\left(x_{1}, x_{2}\right)=-D_{\mathrm{I}}^{\text {self }}\left(x_{2}, x_{1}\right)
$$

Let us first consider $D_{\mathrm{I}}^{\text {self }}\left(x_{1}, x_{2}\right)$. From it we can determine the two-point distribution $T_{\mathrm{I}}^{\text {self }}\left(x_{1}, x_{2}\right)$. From

$$
D_{\mathrm{I}}^{\text {self }}\left(x_{1}, x_{2}\right)=R_{\mathrm{I}}^{\prime}\left(x_{1}, x_{2}\right)-A_{\mathrm{I}}^{\prime}\left(x_{1}, x_{2}\right)
$$




$$
=R_{\mathrm{I}}\left(x_{1}, x_{2}\right)-A_{\mathrm{I}}\left(x_{1}, x_{2}\right)
$$

and

$$
\begin{aligned}
D_{\mathrm{II}}^{\text {self }}\left(x_{1}, x_{2}\right) & =R_{\mathrm{II}}^{\prime}\left(x_{1}, x_{2}\right)-A_{\mathrm{II}}^{\prime}\left(x_{1}, x_{2}\right) \\
& =R_{\mathrm{II}}\left(x_{1}, x_{2}\right)-A_{\mathrm{II}}\left(x_{1}, x_{2}\right)
\end{aligned}
$$

and using (66) we conclude that

$$
\begin{aligned}
& R_{\mathrm{II}}^{\prime}\left(x_{1}, x_{2}\right)=A_{\mathrm{I}}^{\prime}\left(x_{2}, x_{1}\right) ; \\
& A_{\mathrm{II}}^{\prime}\left(x_{1}, x_{2}\right)=R_{\mathrm{I}}^{\prime}\left(x_{2}, x_{1}\right) ; \\
& R_{\mathrm{II}}\left(x_{1}, x_{2}\right)=A_{\mathrm{I}}\left(x_{2}, x_{1}\right) ; \\
& A_{\mathrm{II}}\left(x_{1}, x_{2}\right)=R_{\mathrm{I}}\left(x_{2}, x_{1}\right) .
\end{aligned}
$$

So, we have

$$
T_{\mathrm{I}}^{\mathrm{self}}\left(x_{1}, x_{2}\right)=T_{\mathrm{II}}^{\mathrm{self}}\left(x_{2}, x_{1}\right)
$$

and conclude that the two-point distribution for the self energy $T^{\text {self }}\left(x_{1}, x_{2}\right)=$ $T_{\mathrm{I}}^{\text {self }}\left(x_{1}, x_{2}\right)+T_{\mathrm{II}}^{\text {self }}\left(x_{1}, x_{2}\right)$ is symmetric, as it would be. Moreover it suffices to compute one of the distributions, say $T_{\mathrm{I}}^{\text {self }}\left(x_{1}, x_{2}\right)$, and the other will be given by (67).

Returning to $D_{\mathrm{I}}^{\text {self }}\left(x_{1}, x_{2}\right)$ in (64), we must first verify the causal property of the support of this distribution. The term into brackets can be writen as

$$
S\left(x_{1}-x_{2}\right) D_{0}^{+}\left(x_{2}-x_{1}\right)+S^{+}\left(x_{1}-x_{2}\right) D_{0}\left(x_{1}-x_{2}\right)
$$

and, as $S\left(x_{1}-x_{2}\right)$ and $D_{0}\left(x_{1}-x_{2}\right)$ are causal distributions, so it is also $D_{\mathrm{I}}^{\text {self }}\left(x_{1}, x_{2}\right)$.

The numerical distribution we have to split is

$$
d_{\mathrm{I}}(y)=-e^{2} \beta^{\mu}\left[S^{-}(y) D_{0}^{+}(-y)+S^{+}(y) D_{0}^{+}(y)\right] \beta_{\mu} .
$$


We denote the two terms into brackets in this expression by

$$
d_{-}(y)=S^{-}(y) D^{+}(-y)=-S^{-}(y) D^{-}(y)
$$

and

$$
d_{+}(y)=S^{+}(y) D^{+}(y)
$$

Taking the Fourier transform of $d_{-}$and using the explicit form of Pauli-Jordan distributions we obtain

$$
\widehat{d}_{-}(p)=\frac{1}{(2 \pi)^{4} m} \int d q \not q(\not q+m) \theta\left(-q^{0}\right) \delta\left(q^{2}-m^{2}\right) \theta\left(q^{0}-p^{0}\right) \delta\left[(p-q)^{2}\right]
$$

By virtue of the deltas and thetas, we have that $q^{2}=m^{2}, q^{0}<0,(p-q)^{2}=0$ and $q^{0}-p^{0}>0$. So, $p=(p-q)+q$ is the sum of two 4 -vectors, one timelike and the other lightlike, both in the backward light cone. So, $p$ is timelike. Then we can choose a Lorentz frame such that $p=\left(p^{0}, \overrightarrow{0}\right)$. We first consider the integral proportional to $m$ in the integrand of this expression:

$$
I_{1 \mu}=\int d q q^{\mu} \theta\left(-q^{0}\right) \theta\left(q^{0}-p^{0}\right) \delta\left(q^{2}-m^{2}\right) \delta\left[(p-q)^{2}\right] .
$$

For $\mu \neq 0$ this integral vanishes by symmetry considerations. For $\mu=0$ the integral can be solved in a straightforward manner and results

$$
I_{10}=\frac{\pi}{4} p^{0}\left(1-\frac{m^{4}}{\left(p^{0}\right)^{4}}\right) \theta\left[\left(p^{0}\right)^{2}-m^{2}\right] \theta\left(-p^{0}\right) .
$$

The corresponding term in (71), in any reference frame, is given by

$$
\widehat{d}_{-}(p)_{1}=\frac{1}{(4 \pi)^{3}} \not p\left(1-\frac{m^{4}}{p^{4}}\right) \theta\left(p^{2}-m^{2}\right) \theta\left(-p^{0}\right) \text {. }
$$

Now we calculate the term proportional to $\not^{2}$ in (71):

$$
I_{2 \mu \nu}=\int d q q_{\mu} q_{\nu} \theta\left(-q^{0}\right) \theta\left(q^{0}-p^{0}\right) \delta\left(q^{2}-m^{2}\right) \delta\left[(p-q)^{2}\right] .
$$


Also this integral vanishes for $\mu \neq \nu$ by symmetry reasons. By Lorentz invariance it must be proportional to $g_{\mu \nu}$. The proportionality coefficient is determined by saturation with $g^{\mu \nu}$. Thus

$$
I_{2 \mu \nu}=\frac{1}{4} g_{\mu \nu} \int d q q^{2} \theta\left(-q^{0}\right) \theta\left(q^{0}-p^{0}\right) \delta\left(q^{2}-m^{2}\right) \delta\left[(p-q)^{2}\right] .
$$

This integral can now be easily solved and gets the following contribution to integral (71):

$$
\widehat{d}_{-}(p)_{2}=\frac{m}{2(4 \pi)^{3}} g_{\mu \nu} \beta^{\mu} \beta^{\nu}\left(1-\frac{m^{2}}{p^{2}}\right) \theta\left(p^{2}-m^{2}\right) \theta\left(-p^{0}\right) .
$$

Combining (72) and (73) into (71) we get the final result for $\widehat{d}_{-}(p)=\widehat{d}_{-}(p)_{1}+$ $\widehat{d}_{-}(p)_{2}:$

$$
\widehat{d}_{-}(p)=\frac{1}{(4 \pi)^{3}} \theta\left(p^{2}-m^{2}\right) \theta\left(-p^{0}\right)\left(1-\frac{m^{2}}{p^{2}}\right)\left[\frac{m}{2} \beta^{\mu} \beta_{\mu}+\not p\left(1+\frac{m^{2}}{p^{2}}\right)\right] .
$$

Turning back to (68) we have

$$
\begin{aligned}
-e^{2} \beta^{\mu} \widehat{d}_{-}(p) \beta_{\mu} & =-\frac{e^{2}}{(4 \pi)^{3}} \theta\left(p^{2}-m^{2}\right) \theta\left(-p^{0}\right)\left(1-\frac{m^{2}}{p^{2}}\right)\left[2 m+\not p\left(1+\frac{m^{2}}{p^{2}}\right)\right] \\
& =\widehat{r}_{\mathrm{I}}(p)
\end{aligned}
$$

where we used the following algebraic relations for $\beta$ matrices

$$
\beta^{\mu} \beta^{\nu} \beta_{\mu}=\beta^{\nu}
$$

and

$$
\beta^{\mu} \beta^{\nu} \beta_{\nu} \beta_{\mu}=4
$$

\footnotetext{
${ }^{\dagger}$ We can show that this identity is valid using the usual scalar representation of section 2 . As the r.h.s. is invariant under changes of representation, this result is general, i. e., representation independent.
} 
The distribution $\widehat{d}_{+}(p)$ is calculated in the same way and results

$$
-e^{2} \beta^{\mu} \widehat{d}_{+}(p) \beta_{\mu}=\frac{e^{2}}{(4 \pi)^{3}} \theta\left(p^{2}-m^{2}\right) \theta\left(p^{0}\right)\left(1-\frac{m^{2}}{p^{2}}\right)\left[2 m+\not p\left(1+\frac{m^{2}}{p^{2}}\right)\right] \text {. }
$$

Now, (75) and (76) together give

$$
\widehat{d}_{\mathrm{I}}(p)=\frac{e^{2}}{(4 \pi)^{3}} \theta\left(p^{2}-m^{2}\right)\left(1-\frac{m^{2}}{p^{2}}\right)\left[2 m+\not p\left(1+\frac{m^{2}}{p^{2}}\right)\right] \operatorname{sgn}\left(p^{0}\right) .
$$

We verify that the singular order of this distribution is $\omega=1$. We then split it by using the central splitting formula (31) with $\omega=1$. For $p$ timelike this yelds

$$
\hat{r}_{\mathrm{I}}\left(p^{0}\right)=\frac{i}{2 \pi}\left(p^{0}\right)^{2} \int_{-\infty}^{+\infty} d k^{0} \frac{\hat{d}_{1}\left(k^{0}\right)}{\left(k^{0}-i 0\right)^{2}\left(p^{0}-k^{0}+i 0\right)}
$$

We turn (78) into this integral and solve it by standard methods [21]. For an arbitrary Lorentz frame we arrive at

$$
\begin{aligned}
\hat{r}_{\mathrm{I}}(p) & =\frac{i e^{2}}{4(2 \pi)^{4}} \\
& \times\left\{\left[\log \left|1-b^{2}\right|-i \pi \operatorname{sgn}\left(p^{0}\right) \theta\left(p^{2}-m^{2}\right)\right]\left[m\left(1-\frac{1}{b^{2}}\right)+\frac{p}{2}\left(1-\frac{1}{b^{4}}\right)\right]\right. \\
& \left.-\frac{\not p}{2 b^{2}}-m-\frac{p}{4}\right\},
\end{aligned}
$$

with $b^{2}=\frac{p^{2}}{m^{2}}$. We can analitically extend this result for arbitrary complex $p$ :

$$
\begin{aligned}
\hat{r}_{\mathrm{I}}^{\mathrm{an}}(p)= & \frac{i e^{2}}{4(2 \pi)^{4}}\left\{\log \left(1-b^{2}\right)\left[m\left(1-\frac{1}{b^{2}}\right)+\frac{\not p}{2}\left(1-\frac{1}{b^{4}}\right)\right]\right. \\
& \left.-\frac{\not p}{2 b^{2}}-m-\frac{\not p}{4}\right\},
\end{aligned}
$$

Combining (79) with (75), we obtain $T_{\mathrm{I}}^{\text {self }}\left(x_{1}, x_{2}\right)$ :

$$
T_{\mathrm{I}}^{\text {self }}\left(x_{1}, x_{2}\right)=i e^{2}: \bar{\psi}\left(x_{1}\right) \Sigma\left(x_{1}-x_{2}\right) \psi\left(x_{2}\right):,
$$

where

$$
\begin{aligned}
\widehat{\Sigma}(p)= & -i\left[\widehat{r}_{\mathrm{I}}(p)-\widehat{r}_{\mathrm{I}}(p)\right] \\
= & \frac{i e^{2}}{4(2 \pi)^{4}}\left\{\left[\log \left|1-b^{2}\right|-i \pi \theta\left(p^{2}-m^{2}\right)\right]\left[m\left(1-\frac{1}{b^{2}}\right)+\frac{p}{2}\left(1-\frac{1}{b^{4}}\right)\right]\right. \\
& \left.-\frac{\not p}{2 b^{2}}-m-\frac{\not p}{4}\right\} .
\end{aligned}
$$


This result is free of infrared divergences, because we have used the central splitting formula, which normalizes the solution at the point $p=0$. Also, it is well defined on mass shell. Because $\omega=1$ we must add to this result a first order polynimial in $\not p$ in order to get the general solution to the splitting problem. Thus,

$$
\begin{aligned}
\widetilde{\Sigma}(p)= & \frac{i e^{2}}{4(2 \pi)^{4}}\left\{\left[\log \left|1-b^{2}\right|-i \pi \theta\left(p^{2}-m^{2}\right)\right]\left[m\left(1-\frac{1}{b^{2}}\right)+\frac{p}{2}\left(1-\frac{1}{b^{4}}\right)\right]\right. \\
& \left.-\frac{\not p}{2 b^{2}}+C_{0}+C_{1} \not p\right\} .
\end{aligned}
$$

We now consider radiative corrections due to the self energy insertions into the DKP propagator. The complete propagator is

$$
\widehat{T}_{\text {compl }}^{\mathrm{c}}(p)=\widehat{T}^{\mathrm{c}}(p)+\widehat{T}^{\mathrm{c}}(p) \widetilde{\Sigma}(p) \widehat{T}^{\mathrm{c}}(p)+\widehat{T}^{\mathrm{c}}(p) \widetilde{\Sigma}(p) \widehat{T}^{\mathrm{c}}(p) \widetilde{\Sigma}(p) \widehat{T}^{\mathrm{c}}(p)+\ldots
$$

where $\widehat{T}^{\mathrm{c}}(p)$ is given by (55). Formally summing this series we obtain

$$
\begin{aligned}
\widehat{T}_{\text {compl }}^{\mathrm{c}}(p) & =\frac{\widehat{T}^{\mathrm{c}}(p)}{1-\widetilde{\Sigma}(p) \widehat{T}^{\mathrm{c}}(p)} \\
& =\frac{1}{(2 \pi)^{2} m} \frac{\not p(p+m)-p^{2}+m^{2}}{p^{2}-m^{2}+i 0-\left[\not p(p p+m)-p^{2}+m^{2}\right] \widetilde{\Sigma}(p)} .
\end{aligned}
$$

We require that $m$ be the physical mass of the DKP particle. This will be satisfied only if

$$
\left.\left[\not p(\not p+m)-p^{2}+m^{2}\right] \widetilde{\Sigma}(p)\right|_{p^{2}=m^{2}}=0
$$

Substituting (83) into this condition we obtain the following restriction on the arbitrary constants $C_{0}$ and $C_{1}$ :

$$
C_{0}=m\left(\frac{1}{2}-C_{1}\right) .
$$

This condition eliminates one of the arbitrary constants, say $C_{0}$. The remaining one will be related by an Ward identity to another arbitrary constant that will appear in the vertex distribution. In the Appendix we derive this Ward identity by requiring gauge invariance of the theory. 
Now we calculate the vertex distribution in the limit of zero tansferred momentum by making use of the Ward identity (95) deduced in the Appendix:

$$
\widehat{\Lambda}^{\mu}(p, p)=\frac{1}{(2 \pi)^{2}} \frac{\partial}{\partial p_{\mu}} \widehat{\Sigma}(p) .
$$

Substituting the explicit form of the self-energy (83) into this identity we obtain

$$
\begin{aligned}
\widehat{\Lambda}^{\mu}(p, p)= & \frac{i e^{2}}{4(2 \pi)^{6}}\left\{\left[\log \left|1-b^{2}\right|-i \pi \theta\left(p^{2}-m^{2}\right)\right]\right. \\
& \times\left[\frac{2 p^{\mu}}{m b^{4}}\left(1+\frac{p}{m b^{2}}\right)+\frac{\beta^{\mu}}{2}\left(1-\frac{1}{b^{4}}\right)\right] \\
& +\frac{2 p^{\mu}}{m^{2}}\left[\mathrm{P} \cdot \mathrm{V} \cdot \frac{1}{b^{2}-1}-i \pi \delta\left(b^{2}-1\right)\right]\left[m\left(1-\frac{1}{b^{2}}\right)+\frac{p}{2}\left(1-\frac{1}{b^{4}}\right)\right] \\
& \left.+\frac{p^{\mu} \not p}{m^{2} b^{4}}-\frac{\beta^{\mu}}{2 b^{2}}+C_{1} \beta^{\mu}\right\} .
\end{aligned}
$$

This result is well defined at $p=0$, but it is singular on mass shell $p^{2}=m^{2}$ due to the logarithmic term.

The above form of the vertex function suffices to study the physical meaning of the constant $C_{1}$, which will be done in conection to charge normalization. The physical charge is defined in the scattering of a scalar particle by an external electromagnetic field at low energies. Thus we must consider the contributions (in the limit of zero transferred momentum) to $S$ matrix from the terms containing $C_{1}$ in both self-energy and vertex distributions. Because of the above mentioned mass shell singularity, we must be care in taking the adiabatic limit. Making so, we can prove that all these contributions cancel themselves and conclude that this constant has no physical meaning. Nevertheless, it can be specified by requiring the vertex function to satisfy the central splitting condition, i. e.

$$
\widehat{\Lambda}^{\mu}(0,0)=0
$$

Using this condition into (84) we obtain $C_{1}=\frac{1}{4}$. 


\section{Concluding remarks}

In this paper we have considered scalar QED based on Duffin-Kemmer-Petiau equation in the framework of Epstein-Glaser causal method. We have given the basis to construct the second order $\mathrm{S}$ matrix and calculate the lowest order distributions for Compton scattering, vacuum polarization and self-energy. By using the gauge invariance requirement we determined the vertex correction from the self-energy one.

The starting point of the causal approach was the identification of the onepoint distribution $T_{1}(x)$, which was given by the interaction term in the Lagrangian (13) of the theory, where all the fields entering that expression were free fields. Thus, the causal method specified completely the form of the interaction, giving us a non effective theory. After determining, by using gauge invariance, the finite normalization constant appearing in the two-point distribution for Compton scattering, this distribution was identified with the propagator of the DKP scalar field. Then, in the causal approach we have recovered, in a natural and even simpler way, the basic quantities from which it is constructed the usual effective theory. Namely, we identified $i T^{\mathrm{c}}(x)$ as the effective propagator and $-e$ : $\bar{\psi}(x) \beta^{\mu} \psi(x):(\psi$ and $\bar{\psi}$ being free fields) as the "vertex" (interaction).

At one loop level we have calculated the scalar vacuum polarization tensor, the self-energy and the vertex correction. We have determined all physically meaningful finite normalization constants from physical requirements as symmetries and mass and charge normalization. Our results agree with that obtained in the context of the effective theory. To do a complete analysis at one loop level, we would have to calculate as well the singular order of the four point distribution for scattering of two scalar particles. This problem is fundamentally important to study the renormalizability of the theory and is under our investigation presently. 
As future perspectives we can quote the use of the causal approach to study DKP field interacting with external gravitational fields.

\section{Acknowlegdments}

J.T.L. and B.M.P. thank respectively to CAPES-PICDT and CNPq for partial support. L.A.M and J.S.V. thank FAPESP for full support.

\section{Appendix: gauge invariance}

The gauge invariance requirement allows us to determine the remaining arbitrary constant $C$, in the two-point distribution for Compton scattering, and to find a relation (Ward identity) between the self-energy and vertex distributions, thus relating the corresponding arbitrary constants.

As we saw, in the causal approach all the fields are free fields. Then, under a gauge transformation the electromagnetic field transforms as $A_{\mu}(x) \rightarrow A_{\mu}(x)+$ $\partial_{\mu} \Lambda(x)$, whereas the matter fields $\psi(x)$ and $\bar{\psi}(x)$ remains unaffected. Here $\Lambda(x)$ is a c-number scalar field that satisfies $\square \Lambda(x)=0$ (Lorentz gauge) and vanishes at infinity.

The gauge invariance requirement amounts that in the adiabatic limit, $g \rightarrow 1$, the $\mathrm{S}$ matrix must be invariant under such transformations, which implies the invariance of all the terms in the $\mathrm{S}$ matrix expansion (16). We write a generic $n$-point distribution normally ordered with respect to photon operators in the form

$$
T_{n}\left(x_{1}, \ldots, x_{n}\right)=\sum_{l=0}^{n} \sum_{1 \leq k_{1}<\ldots<k_{l} \leq n} t_{k_{1} \ldots k_{l}}^{\mu_{1} \ldots \mu_{l}}\left(x_{1}, \ldots, x_{n}\right): A_{\mu_{1}}\left(x_{k_{1}}\right) \ldots A_{\mu_{l}}\left(x_{k_{l}}\right):
$$

where $t_{k_{1} \ldots k_{l}}^{\mu_{1} \ldots \mu_{l}}\left(x_{1}, \ldots, x_{n}\right)$ contains only scalar operators and it is the sum of all 
graphs of order $n$ with only $l$ external photon lines, attached at the vertices $x_{k_{1}}, \ldots, x_{k_{l}}$. The external scalar operators are arbitrary. Applying the gauge invariance requirement we arrive at the following condition [21]:

$$
\frac{\partial}{\partial x_{k_{j}}^{\mu_{j}}} t_{k_{1} \ldots k_{l}}^{\mu_{1} \ldots \mu_{l}}\left(x_{1}, \ldots, x_{n}\right)=0,
$$

for all $1 \leq l \leq n$, all $1 \leq j \leq l$, all $1 \leq k_{1}<\ldots<k_{l} \leq n$ and all $\left(x_{1}, \ldots, x_{n}\right) \in \mathbf{R}^{4 \mathbf{n}}$.

When we apply this condition for the two-point Compton distribution, we get at the condition

$$
\partial_{\mu}^{1} Q^{\mu \nu}\left(x_{1}, x_{2}\right)=0
$$

where

$$
\begin{aligned}
Q^{\mu \nu}\left(x_{1}, x_{2}\right)= & : \bar{\psi}\left(x_{1}\right) \beta^{\mu} t^{\mathrm{I}}\left(x_{1}, x_{2}\right) \beta^{\nu} \psi\left(x_{2}\right): \\
& +: \bar{\psi}\left(x_{2}\right) \beta^{\nu} t^{\mathrm{II}}\left(x_{1}, x_{2}\right) \beta^{\mu} \psi\left(x_{1}\right): \\
= & Q^{\nu \mu}\left(x_{2}, x_{1}\right) .
\end{aligned}
$$

and $\partial_{\mu}^{1}$ means derivative with respect to $x_{1}$.

Turning (87), (49) and (51) into this last equation, the l.h.s. gives

$$
\begin{aligned}
\partial_{\mu}^{1} Q^{\mu \nu}\left(x_{1}, x_{2}\right)= & -: \partial_{\mu} \bar{\psi}\left(x_{1}\right) \beta^{\mu}\left[S^{\mathrm{F}}\left(x_{1}-x_{2}\right)-C \delta\left(x_{1}-x_{2}\right)\right] \beta^{\nu} \psi\left(x_{2}\right): \\
& -: \bar{\psi}\left(x_{1}\right) \beta^{\mu}\left[\partial_{\mu} S^{\mathrm{F}}\left(x_{1}-x_{2}\right)-C \partial_{\mu} \delta\left(x_{1}-x_{2}\right)\right] \beta^{\nu} \psi\left(x_{2}\right): \\
& -: \bar{\psi}\left(x_{2}\right) \beta^{\nu}\left[-\partial_{\mu} S^{\mathrm{F}}\left(x_{2}-x_{1}\right)+C \partial_{\mu} \delta\left(x_{2}-x_{1}\right)\right] \beta^{\mu} \psi\left(x_{1}\right): \\
& -: \bar{\psi}\left(x_{2}\right) \beta^{\nu}\left[S^{\mathrm{F}}\left(x_{2}-x_{1}\right)-C \delta\left(x_{2}-x_{1}\right)\right] \beta^{\mu} \partial_{\mu} \psi\left(x_{1}\right): .
\end{aligned}
$$

From the equation of scalar Feynman propagator

$$
\left(\square+m^{2}\right) \triangle^{\mathrm{F}}(x)=\delta(x)
$$

the relation (4) and definition (50), we have

$$
i \not \partial S^{\mathrm{F}}(x)=m S^{\mathrm{F}}(x)+\frac{i}{m} \not \partial \delta(x) .
$$


Using this into (88), condition (86) yields

$$
C=\frac{I}{m}
$$

where $I$ is the $5 \times 5$ identity matrix.

Now we consider the three-point distributions corresponding to the vertex correction. We must consider four graphs, that are analogous to that we find in the vertex correction of spinor QED. Considering the photon line attached at the vertex $x_{3}$, the distribution $t_{3}^{\mu}\left(x_{1}, x_{2}, x_{3}\right)$ we must enter into condition (85) is

$$
\begin{aligned}
t_{3}^{\mu}\left(x_{1}, x_{2}, x_{3}\right)= & -: \bar{\psi}\left(x_{1}\right) \Lambda^{\mu}\left(x_{1}-x_{3}, x_{2}-x_{3}\right) \psi\left(x_{2}\right): \\
& +: \bar{\psi}\left(x_{1}\right) \Sigma\left(x_{1}-x_{2}\right) T^{\mathrm{c}}\left(x_{2}-x_{3}\right) \beta^{\mu} \psi\left(x_{3}\right): \\
& +: \bar{\psi}\left(x_{3}\right) \beta^{\mu} T^{\mathrm{c}}\left(x_{3}-x_{1}\right) \Sigma\left(x_{1}-x_{2}\right) \psi\left(x_{2}\right): \\
& +: \bar{\psi}\left(x_{1}\right) \beta^{\rho} \psi\left(x_{1}\right): g_{\rho \nu} D_{0}^{\mathrm{F}}\left(x_{1}-x_{2}\right) \Pi^{\nu \mu}\left(x_{2}-x_{3}\right) \\
& +x_{1} \longleftrightarrow x_{2}
\end{aligned}
$$

where $T^{\mathrm{c}}$ is the "effective DKP propagator" given in (53). Thus we have

$$
\begin{aligned}
\partial_{\mu}^{3} t_{3}^{\mu}\left(x_{1}, x_{2}, x_{3}\right)= & : \bar{\psi}\left(x_{1}\right)\left[-\partial_{\mu}^{3} \Lambda^{\mu}\left(x_{1}-x_{3}, x_{2}-x_{3}\right)\right] \psi\left(x_{2}\right): \\
& +: \bar{\psi}\left(x_{1}\right) \Sigma\left(x_{1}-x_{2}\right) \partial_{\mu}^{3}\left[T^{\mathrm{c}}\left(x_{2}-x_{3}\right) \beta^{\mu} \psi\left(x_{3}\right)\right]: \\
& +: \partial_{\mu}^{3}\left[\bar{\psi}\left(x_{3}\right) \beta^{\mu} T^{\mathrm{c}}\left(x_{3}-x_{1}\right)\right] \Sigma\left(x_{1}-x_{2}\right) \psi\left(x_{2}\right): \\
& +: \bar{\psi}\left(x_{1}\right) \beta^{\rho} \psi\left(x_{1}\right): g_{\rho \nu} D_{0}^{\mathrm{F}}\left(x_{1}-x_{2}\right)\left[\partial_{\mu}^{3} \Pi^{\nu \mu}\left(x_{2}-x_{3}\right)\right] \\
& +x_{1} \longleftrightarrow x_{2} .
\end{aligned}
$$

For the brackets in the second and third lines we have

$$
\begin{aligned}
\partial_{\mu}^{3}\left[T^{\mathrm{c}}\left(x_{2}-x_{3}\right) \beta^{\mu} \psi\left(x_{3}\right)\right] & =i \delta\left(x_{2}-x_{3}\right) \psi\left(x_{3}\right) \\
\partial_{\mu}^{3}\left[\bar{\psi}\left(x_{3}\right) \beta^{\mu} T^{\mathrm{c}}\left(x_{3}-x_{1}\right)\right] & =-i \bar{\psi}\left(x_{3}\right) \delta\left(x_{3}-x_{1}\right)
\end{aligned}
$$

where we have used the DKP equation and the fact that $T^{\mathrm{c}}$ is the corresponding DKP Green function (see eq. (54)). Turning these results into (91) and taking 
into account the delta distributions, we obtain

$$
\begin{aligned}
\partial_{\mu}^{3} t_{3}^{\mu}\left(x_{1}, x_{2}, x_{3}\right)= & : \bar{\psi}\left(x_{1}\right)\left\{-\partial_{\mu}^{3} \Lambda^{\mu}\left(x_{1}-x_{3}, x_{2}-x_{3}\right)\right. \\
& \left.+i \delta\left(x_{2}-x_{3}\right) \Sigma\left(x_{1}-x_{2}\right)-i \delta\left(x_{1}-x_{3}\right) \Sigma\left(x_{1}-x_{2}\right)\right\} \psi\left(x_{2}\right): \\
= & 0 .
\end{aligned}
$$

This condition will be satisfied only if the term into curl brackets vanish. Thus we arrive to the desired Ward identity:

$$
-\partial_{\mu}^{3} \Lambda^{\mu}\left(x_{1}-x_{3}, x_{2}-x_{3}\right)+i \delta\left(x_{2}-x_{3}\right) \Sigma\left(x_{1}-x_{2}\right)-i \delta\left(x_{1}-x_{3}\right) \Sigma\left(x_{1}-x_{2}\right)=0
$$

Denoting

$$
y_{1}=x_{1}-x_{3} \quad \text { and } \quad y_{2}=x_{2}-x_{3},
$$

we can write

$$
\frac{\partial}{\partial x_{3}^{\mu}} \Lambda^{\mu}\left(y_{1}, y_{2}\right)=-\left(\partial_{1}+\partial_{2}\right)_{\mu} \Lambda^{\mu}\left(y_{1}, y_{2}\right)
$$

and equation (93) becomes

$$
\left(\partial_{1}+\partial_{2}\right)_{\mu} \Lambda^{\mu}\left(y_{1}, y_{2}\right)+i \delta\left(y_{2}\right) \Sigma\left(y_{1}-y_{2}\right)-i \delta\left(y_{1}\right) \Sigma\left(y_{1}-y_{2}\right)=0
$$

Taking the Fourier transform of this equation we arrive at

$$
\left(p_{1}+p_{2}\right)_{\mu} \widehat{\Lambda}^{\mu}\left(p_{1}, p_{2}\right)-\frac{1}{(2 \pi)^{2}}\left\{\widehat{\Sigma}\left(p_{1}\right)-\widehat{\Sigma}\left(p_{2}\right)\right\}=0
$$

Introducing the notation $q=-p_{2}, p=p_{1}$ and redefining $\widehat{\Lambda}^{\mu}$ as

$$
\widehat{\Lambda}^{\mu}(p,-q) \rightarrow \widehat{\Lambda}^{\mu}(p, q),
$$

\footnotetext{
$\ddagger$ Our convention to the Fourier transform of a distribution $F\left(y_{1}, y_{2}\right)$ is

$$
\widehat{F}\left(p_{1}, p_{2}\right)=\frac{1}{(2 \pi)^{4}} \int d y_{1} d y_{2} \mathrm{e}^{i\left(p_{1} y_{1}+p_{2} y_{2}\right)} F\left(y_{1}, y_{2}\right)
$$
}


we arrive at

$$
(p-q)_{\mu} \widehat{\Lambda}^{\mu}(p, q)=\frac{1}{(2 \pi)^{2}}\{\widehat{\Sigma}(p)-\widehat{\Sigma}(q)\} .
$$

Taking the limit $p \rightarrow q$ we have

$$
\widehat{\Lambda}^{\mu}(p, p)=\frac{1}{(2 \pi)^{2}} \frac{\partial}{\partial p_{\mu}} \widehat{\Sigma}(p),
$$

which is the form of the Ward identity we use to determine the vertex correction in Section 7.

\section{References}

[1] C. Itzykson and J. B. Zuber, Quantum Field Theory (Mc-GrawHill, Singapore, 1980).

[2] G. Petiau, Acad. R. Belg. Cl. Sci. Mém. Collect 8 16, No. 2 (1936).

[3] N. Kemmer, Proc. Roy. Soc. A 166 (1938) 127.

[4] R. J. Duffin, Phys. Rev. 54 (1938) 1114.

[5] N. Kemmer, Proc. Roy. Soc. A 173 (1939) 91.

[6] H. Umezawa, Quantum Field Theory (North-Holland, Amsterdan, 1956).

[7] N. N. Bogoliubov and D. V. Shirkov, Introduction to the Theory of Quantized Fields (Wiley, New York, 1980).

[8] J. T. Lunardi, B. M.Pimentel, R. G. Teixeira and J. S. Valverde, Phys. Lett. A 268 (2000) 165.

[9] A. I. Akhiezer and V. B. Berestetskii, Quantum Electrodynamics (Interscience, New York, 1965). 
[10] R. A. Krajcik and M. M. Nieto, Am. J. Phys. 45 (1977) 818.

[11] V. Ya. Fainberg and B. M. Pimentel. Phys. Lett. A 271 (2000) 16.

[12] B. C. Clark, S. Hama, G. R. Kälbermann, R. L. Mercer and L. Ray, Phys. Rev. Lett. 55 (1985) 592.

[13] E. Friedman, G. Kälberman and C. J. Batty, Phys. Rev. C 34 (1986) 2244.

[14] M. Nowakowski, Phys. Lett. A 244 (1998) 329.

[15] J. T. Lunardi, B. M. Pimentel and R. G. Teixeira, "Duffin-Kemmer-Petiau equation in Riemannian Space-times" in "Proceedings of Workshop on Geometrical Aspects of Quantum Fields," A. A. Bytsenko, A. E. Gonçalves and B. M. Pimentel (eds.), (World Scientific, Singapore, 2001), p. 111.

[16] V. Ya. Fainberg and B. M. Pimentel, Theor. Math. Phys. 124 (2000) 445.

[17] V. Gribov, Eur. Phys. J. C 10 (1999) 71.

[18] L. K. Kerr, B. C. Clark, S. Hama, L. Ray, and G. W Hoffmann, Prog. Theor. Phys. 103 (2000) 321.

[19] M. Dutsch, F. Krahe and G. Scharf, Il Nuovo Cim. A 106 (1993) 277.

[20] H. Epstein and V. Glaser, Ann. Inst. H. Poincaré A 19 (1973) 211.

[21] G. Scharf, Finite Quantum Electrodynamics: the Causal Approach, $2^{\text {nd }}$ ed. (Springer, Berlin, 1995).

[22] J. Géhéniau, Acad. R. Belg. Cl. Sci. Mém. Collect 8 18, No. 1 (1938).

[23] A. Aste, Ann. Phys. 257 (1997) 158. 
[24] G. Scharf, W. F. Wreszinski, B. M. Pimentel and J. L. Tomazelli, Ann. Phys. 231 (1994) 185.

[25] L. A. Manzoni, B. M. Pimentel and J. L. Tomazelli, Eur. Phys. J. C8 (1999) 353; ibid. 12 (2000) 701.

[26] T. Kinoshita, Progr. Theor. Phys. 3 (1950) 473.

Harish-Chandra, Proc. Roy. Soc. A 186 (1946) 502. 